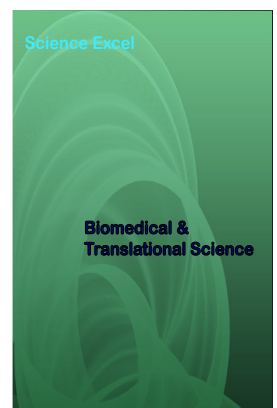

Correspondence

Wolfgang Mastnak,

Professor Dr, Beijing Normal University / Research Centre for Arts Therapies, Hochschule für Musik, Arcisstraße 12, 80333 München, Germany

Orcid.org/0000-0003-4632-5639

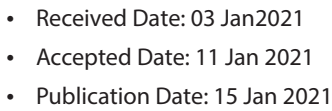

Keywords: Arts Therapies, China, Psycho oncology, Theory of Science, Translational Oncology

Copyright

(C) 2021 Science Excel. This is an openaccess article distributed under the terms of the Creative Commons Attribution 4.0 International license.

\title{
Chinese Arts-Based Psycho-Oncology: A Translational Systemic Meta-Synthetic Perspective
}

\author{
Wolfgang Mastnak*, and Qi Mao \\ Beijing Normal University / Research Centre for Arts Therapies, Hochschule für Musik, Germany
}

\begin{abstract}
Psycho-oncology is a quite new discipline in China. Related arts-based approaches significantly involve translational sciences. From a meta-theoretical and epistemological perspective both use pragmatic inferential reasoning alongside systemic meta-syntheses to scientifically substantiate practical models, here particularly for psycho-oncological treatment in clinical and public health areas. This concerns (i) pathological mental conditions as risk factors of cancer and mortality, (ii) psychiatric squeal of cancer, associated changes of the patient's cognitive and affective states, as well as self-images and socio-cultural inclusion and (iii) mental issues of and social support for family members and caregivers of cancer patients.
\end{abstract}

As concerns underlying mechanisms neuro-oncological, psycho-immunological and epigenetic findings contribute to a multimodal theoretical framework, which includes microbiological, psychological, sociocultural and aesthetic perspectives. Emphasising that efficient and individualised psycho-oncology in practice is inextricably linked with the patient's cultural and a spiritual background, culturally sensitive psycho-oncology is regarded as an overall key principle and not only as a sub discipline. In this context, music therapy using the traditional Chinese cither Guqin as well as creative and explorative work with the patient's voice not only provide mindful arts-based models of psycho-oncology but also encourage other cultures to explore the psycho-oncological potential of their traditional and contemporary arts.

\section{Introduction: China's Breakthrough in Psycho-Oncology.}

Jimmie Coker Holland [1] (1928 - 2017) was a founder of psycho-oncology and an explicit authority on history of psychooncology. At the beginning of the new millennium [2] she looked back to the formal beginnings of psycho-oncology in the mid1970 s, when 'the stigma making the word "cancer" unspeakable was diminished to the point that the diagnosis could be revealed and the feelings of patients about their illness could be explored for the first time'.

In the same breath she pointed out that 'a second stigma has contributed to the late development of interest in the psychological dimensions of cancer: negative attitudes attached to mental illness and psychological problems, even in the context of medical illness' - and these are issues we also find in China today. Elucidating relevant factors she emphasises the broad spectrum of psychooncology: 'behavioural research in changing lifestyle and habits to reduce cancer risk; study of behaviours and attitudes to ensure early detection; study of psychological issues related to genetic risk and testing; symptom control (anxiety, depression, delirium, pain, and fatigue) during active treatment; management of psychological squeal in cancer survivors; and management of the psychological aspects of palliative and end-oflife care'.

New disciplines in medicine are often confronted with obstacles and difficulties, and pioneers of psycho-oncology [3] pointed out what is also in China of crucial relevance and challenging: more attention to and research into the care of the total patient, i.e. the physical, psychological, social and spiritual aspects of care; enhanced quality of life across the continuum of care from diagnosis to palliative care; cancer prevention and early detection and changing attitudes and behaviours that put people at greater risk. And in a later historical review of psycho-oncology, Jimmie C. Holland [4] emphasised a core topic of the present article: 'cross cultural studies that will examine differences in prevention and detection, health care systems, alternative therapies and Meta analyses'. 
Today, psycho-oncology has become an important domain and a study from the department of psychiatry of the Weill Medical College of Cornell University [5] summarises that psychosocial and psychiatric squeal of cancer are highly prevalent, diverse, and challenging for clinicians to manage. The authors emphasised that these squeal may complicate the course of cancer, hence the need for social workers, psychologists, and psychiatrists who can provide consultative services in support of the psychiatric care of cancer patients and their families at all stages of disease, including cancer survivorship.

Since the introduction of oncological social psychology in China in the 1990s medical humanities have been in the ascendant. Both scientific research and clinical practice cast light on the importance of culturally sensitive approaches and the Chinese Cancer Psychology Committee and the AntiCancer Association provide an interactive academic platform for professionals in cancer psychology. Analysing these developments from the perspective of history of medicine, we still discern a major breakthrough this year, hence our suggestion to regard 2020 as the year of birth of Chinese psycho-oncology.

On the $14^{\text {th }}$ of November 2020, the 2020 Annual Meeting of Chinese Oncology Psychology was held in Guangzhou, after Shanghai and Beijing China's third biggest metro pole, northwest of Hong Kong. Hosted by the Cancer Prevention and Treatment Centre of Sun Yat-sen University and the Guangdong Anti-cancer Association, and co-organised by the international Anti-Cancer Union and the Chinese Academy of Integrated Medicine Development Strategy, it was dedicated to 'Humanities, Innovation, Sharing - Promoting Comprehensive Psychosocial Support', and that is what we may call the cradle of advanced Chinese psycho-oncology.

The director of the Chinese association of tumour psychology, Xiao Hong Liu, emphasised the importance of AI-applications for psychological diagnosis and treatment of tumour patients, and pointed out that recognition of the enormous amount of available data is challenging, but for advanced prevention and optimised treatment systems imperative. His information-theoretical approach complements the more humanistic spirit of the present article, particularly when it comes to arts-based interventions.

The framework of Chinese psycho-oncology involves theories and practices from international psycho-oncology and according to Lili Tang, director of the Rehabilitation Department of Peking University Cancer Hospital, the 2020 edition of Chinese guidelines for clinical practice of oncology is considered a cornerstone of advanced psychosocial oncology in mainland China: in her words 'a new era of relevant standards'. Guidelines need implementation and Xiaomei Li from the PLA General Hospital discussed practical ways of how to manage mind full support alongside his suggestion to integrate symptom management and the complex of diagnosis and treatment. Motto: treating both the symptoms and the roots instead of 'taking care of one and losing the other'.

In clinical fields which combine physiological and psychological approaches, the issue of asymmetric communication plays a crucial role and terms such as psycho education in psychiatric [6-8] and/or mindfulness in oncological [9] care come into play. Particularly with regard to breast cancer, Pilin Wang from the Beijing Tiantan Hospital (affiliated to the Capital Medical University) discussed issues of asymmetric communication technology. He claimed that doctors should not only pay attention to the curative effect of the patients' physical treatment, but also to the feelings of patients and their accompanying families and coined the term of the patients' 'strength of rebirth'. Akin to these perspectives, Wanmin Qiang from the Cancer Hospital of Tianjin Medical University highlighted the importance of 'dignified care' and explored related facets of 'life and death education'.

Chinese psycho-oncology faces a dual challenge: on the one hand to make use of successful approaches of the West and on the other to create a genuine Chinese way of psycho-oncology - and this goes hand in hand with one of the main objectives of the present article: culturally sensitive and arts-based psycho-oncology for China. This also concerns culturally responsive attitudes towards pain and referring to the '2018 WHO guidelines for the management of cancer pain in adults and adolescents' Jinxiang Li from the 4th West China Hospital of Sichuan University emphasised that cancer pain is closely related to tumour psychology, and medical staff specialising in the complex area of oncological rehabilitation and psychology, including cancer pain management, is needed.

\section{Psycho-Oncology: Translational Science in Dynamic Systems}

Broadly speaking, translational medicine and translational medical science 'translate' novel discoveries in biological sciences to improve human health and clinical practice, and the famous 'bench-to-bedside' approach is complemented by 'bedside-to-bench' studies providing feedback about the applications of new treatments and how they can be improved. Rubio [10] shed light on the history of the term and found that although a Medline search indicates that 'translational research' appeared as early as 1993, there were relatively few references to it in the medical literature during the 1990s. In the beginning of translational sciences most references are to research about cancer and there are significant contributions to translational epidemiology in cancer medicine [11].

From an epistemological and meta-theoretical perspective translational science and translational medicine can be considered a pragmatic form of inferential reasoning and similarities with inferential statistics in evidence based medicine spring to mind. Nonetheless, from the perspective of theory of science, there are crucial differences: Translational sciences tend to meticulously scrutinise the reliability of their translational reasoning, while evidence based medicine tends to trust standardised research designs, hence their epistemological fragility. For instance, randomisation is not necessarily a reliable means to generate two equivalent samples and it cannot be taken for granted that mathematical distributions 'behave' in the same way as the groups of patients they intend to represent. And although psychological tests are 'validated' they still might fail to identify specific pathological features such as typical anxieties in Chinese breast cancer patients. Broadly speaking, medical research requires metatheoretical effort to explore epistemological reliability and validity and blind belief such as in formal rules of standardised 
research designs are a common trap in medical research.

Additionally to these modes of pragmatic inferential reasoning, translational sciences are outstandingly good at synthesising singular data to create novel theoretical frameworks and it is very likely that this is a main reason why translational medicine has so rapidly gained ground. Research designs of conventional evidence based medicine such as RCTs generate data about effects and their statistical trustworthiness, but they neither explore mechanisms nor do they contribute to more complex epistemological knowledge. In other words, they yield myriads of singular findings but fail in generating new theories. Akin to the translational generation of holistic models, the concept of systemic meta-synthesis [12] provides methods to synthesise singular findings and suggests the novel notion of 'powered hypotheses', which are epistemologically stronger than results from common meta-syntheses. There are striking similarities between research practices of translational sciences and systemic meta-syntheses and further metatheoretical research will yield deeper insights.

While usually evidence based medicine does not discuss problems of transformation between the human domain of patients and the figures of corresponding distributions and their parameters - this is a key topic of the correspondence theory of truth - translational sciences explicitly refer to the issue of qualitative transitions, which also play a crucial role in Chinese arts-based psycho-oncology, e.g. when we discuss possible inter-relationships between the mystical sound of the traditional Chinese cither Guqin, alleviation of obsessivecompulsive thoughts, and tumour markers in breast cancer patients. Moreover, and this is also a significant difference to conventional evidence based medicine, translational sciences take the dynamics of relevant systems into account and this coincides with basic premises of this article: the dynamic interdependencies of corresponding biological, mental, social and cultural worlds.

\section{Core Issues of Culturally Sensitive Psycho-Oncology}

Broadly speaking, there are two main issues in psychooncology, one how the cancer influences the psyche and the other how mental conditions influence the neoplasm. Between these two cornerstones there lies a broad spectrum of problems such as psycho-oncological differences between cancer types or dynamic interdependencies between spiritual attitudes, sociocultural inclusion and oncological epigenetics. In this article we explicitly draw attention to culturally sensitive psycho-oncology which should not be considered a subdiscipline of psycho-oncology but rather one of its essential principles.

\section{Cancer to Psyche}

Reviewing the relevant literature we assume that until now the lion's share of studies focus on the impact of cancer on the psyche, and it is very likely that his has two reasons: a historical and a methodological one. As mentioned above, founding psycho-oncology as a discipline was much inspired by the phenomenon that the life-event 'cancer' - including treatment, cognitive changes and social issues - may considerably impact on the patient's mental health and personality, hence the need for specific support. The methodological reason goes hand in hand with a relatively easy discovery of mental health issues such as anxiety and depression in oncological patients, and evidence based medicine provides clear-cut research designs.

Depressive responses to cancer are totally understandable and well substantiated by psycho-oncological studies: Krebber [13] suggested a pooled mean prevalence of depression in cancer patients from $8 \%$ to $24 \%$ which differs by the type of instrument, type of cancer and treatment phase, an Italian review [14] speaks of a prevalence of depression and depressive disorders between $5 \%$ and $60 \%$ according to different diagnostic criteria, different research tools, as well as the stage and type of cancer, and a Greek research group [15] concluded that breast cancer patients - this is also the clientele of a current research on music therapy in China - are in high risk for developing psychiatric disorders such as depression and anxiety.

In this context the question arises whether depression is rather a squeal of the mental awareness of cancer or if there is also mutual influence between depression and cancer, and this is the core issue of the next sub-chapter. A meta-analysis [16] with a total of 1469179 participants and 89716 incident cases of cancer from 25 studies concluded that depression was significantly associated with overall cancer risk, and compatible with this result a German meta-analysis on depression and cancer mortality [17] found that depression diagnosis and higher levels of depressive symptoms predicted elevated mortality. Particularly in line with our hypothesis of dynamic interdependencies between mental and oncological states the study highlights that 'this was true in studies that assessed depression before cancer diagnosis as well as in studies that assessed depression following cancer diagnosis'.

In this context the biology of depression in cancer and the relationship between depression and cancer progression plays a crucial role and Jorge Luis Sotelo [18] summarised that the 'prevalence of depressive symptoms in patients with cancer exceeds that observed in the general population and depression is associated with a poorer prognosis in cancer patients. The increased prevalence is not solely explained by the psychosocial stress associated with the diagnosis. Proinflammatory cytokines, which induce sickness behaviour with symptoms overlapping those of clinical depression, are validated biomarkers of increased inflammation in patients with cancer ... chronic inflammatory processes associated with stress may also underlie depression symptoms in general, and in patients with cancer in particular'.

Depression in cancer patients if often combined with anxiety [19] and together they influence quality of life, adherence to treatment, cancer survival, risk of suicide and treatment costs. A Canadian study [20] analysed prevalence rates of anxiety and depression after cancer diagnosis by cancer type, gender and age and concluded that across cancer types $19.0 \%$ of patients showed clinical levels of anxiety and another $22.6 \%$ had subclinical symptoms. $12.9 \%$ of patients reported clinical symptoms of depression and an additional $16.5 \%$ described subclinical symptoms. Analyses by cancer type revealed significant differences and patients with lung, gynaecological or haematological cancer reported the highest levels of distress at the time point of cancer diagnosis. In this sample, women showed higher rates of anxiety and depression, and for some cancer types the prevalence was two to three times higher than that seen for men.

Particularly in advanced cancer demoralisation and death 
anxiety are likely to torture patients [21] and - from a Chinese perspective - spiritual backgrounds such as Buddhism, Daoism or animistic traditions of the various ethnicities living in China become important factors of psycho-oncology. Concerning anxiety in cancer patients, an Australian research group [22] identified already existing relevant theories and proposed an integrated model of cancer-related anxiety, and this is akin to how we develop our Chinese arts-based psychooncological approach. They identified nine theoretical models of anxiety in the context of cancer, isolated five core topics pre-existing schema, the inherent nature of cancer, cognitive factors, coping responses and contextual factors - and set up a theoretical framework to explain the development and maintenance of anxiety in the context of cancer suggesting 'that pre-existing schema, past experiences of cancer, an intolerance of uncertainty and meta-cognitive beliefs about worry interact with the inherent nature of cancer to produce overwhelming distress. The distress activates cognitive processes characterized by vigilance, worry and rumination. Attempts to cope by re-establishing control, and a pattern of vigilance to cancer-related cues and/or avoidance reinforce anxiety, in the context of a range of systemic factors that can either buffer against or worsen the anxiety'.

Psychopathological and psychiatric issues in psychooncology cover a broad spectrum and call for specific diagnosis- and personality-centred intervention. Findings about body image difficulties in cancer patients [23] are compatible with own clinical observations, and sexual health care has become an important domain in translational psychooncology including issues such as sexual quality of life in cancer survivors [24]. Such problems also relate to the adverse impact of oestrogen deprivation and therapeutic interventions such as radiation, chemotherapy and surgery on libido, sexual arousal, orgasmic function and the ability to have pleasurable intercourse in post-breast cancer patients [25], a problem which immensely depends on sociocultural attitudes towards sexuality, China included. Similarly sexual issues in palliative care still sound weird in some cultures, although they might be of high individual importance and hence a topic in psychooncology [26] - and arts therapies can help to increase openminded attitudes towards this sensitive area.

The more we enter individualised and narrative psychooncology, the more we become aware of the importance of culturally sensitive mindfulness in this domain. Particularly newer studies draw attention to the important role spirituality may play in cancer patients [27] and Portuguese researchers [28] showed that spirituality can support posttraumatic growth in breast cancer patients. In this context, China's multifaceted spiritual landscape [29] including Buddhism, Daoism, traditional forms of animism, Christianity, Islam, atheism etc. gains in importance and involves cultural and arts-based practices.

Also for the patient's family cancer is a serious and life disturbing event. Caregivers of cancer patients are confronted with high emotional pressure and complex challenges, and the authors' Chinese research particularly focuses on the patients' spouses. For thousands of years, Chinese thinking has deeply been influenced by Confucian culture and thought - in Chinese 儒家 Rújiā - and the concept of 'family' is even today inextricably linked with the peoples' minds. Malignant tumours of family members tend to have a strong impact on the whole family: emotionally, cognitively and behaviourally. A case review [30] suggests that especially in cases of advanced malignancies couple therapy can help to manage differences in emotional interaction, and benefits of emotional support in couples facing end-stage cancer are evident. A study on early breast cancer [31] showed that couple-oriented adjustment and couple-based interventions are potentially efficient treatment options for pairs who experience profound, relationship-associated pain following diagnosis and treatment of breast cancer. In oncological domains artsbased therapies are promising but both in science and practice still underrepresented approaches - a reason why the second author of the present article conducts research on Chinese arts-based psycho-oncology.

\section{Psyche to Cancer}

The second issue is more controversially discussed and Naoki Nakaya [32] concluded that effects of personality traits and depression on cancer risk and survival appear to be 'extremely small'. Reviewing relevant articles surprises with a huge number of studies suggesting that psychological conditions such as depression did not increase the risk of cancer and the results of a Korean meta-analysis [33] did not demonstrate that people with depressive disorder are at increased risk for developing cancer. Taking contradictory evidence based outcomes as well as findings about underlying mechanisms of cancer and mental issues into account, we assume epistemological and meta-methodological errors in research which categorically denies interdependencies between psychological and oncological processes.

Focusing on the impact of social isolation on cancer, Lutgendorf [34] highlighted that 'noradrenergic pathways have been implicated in growth and progression of ovarian cancer', low subjective social support was associated with elevated intratumoral norepinephrine, beta-adrenergic signalling is related to key biological pathways involved in tumor growth, and that these findings may have implications for patient outcomes in ovarian cancer. This is not only of considerable importance when it comes to social distancing due to pandemic measures such as in the COVID-19 era, it also is of high relevance to arts-based psycho-oncological group activities.

In contrast to evidence based studies which speak of weak or no significant causal connections between psychosocial factors and cancer, a more systemic and interdisciplinary view calls for further research. A Slovakian study [35] refers to findings that 'the nervous system, via neural and humoral pathways, significantly modulates processes related to cancer at the level of the tumor micro- and macro environments' and that 'data accumulated in the last two decades have clearly shown that effects of the nervous system on cancer initiation, progression, and the development of metastases are mediated by the sympathoadrenal system mainly via $\beta$-adrenergic receptor signalling' and provide a 'new complex view of the role of $\beta$-adrenergic receptor signalling within the tumor micro- and macro environments as well as in mediating the effects of the psychosocial and spiritual environments'.

In this interdisciplinary domain, Lamboy-Caraballo [36] pointed out that psychological distress in epithelial ovarian 
cancer patients is not only associated with worse quality of life and poor treatment adherence but may also influence chemotherapy response and prognosis, and taking relevant facts together they concluded that stress hormones can affect DNA integrity and modulate cisplatin resistance in epithelial ovarian cancer cells. Consistent with such views, we greatly suggest multidimensional and interdisciplinary approaches to discover (possible) psycho-biological systems influencing cancer, and this is akin to studies on multi-systemic biological risks (MSBR) of cancer [37].

Without downplaying the importance of evidence based medicine in the sense of designs such as RCTs, we assume a certain risk of overlooking psychosocial and psychomicrobiological carcinogenic factors in mere input-outcomebased designs, which also believe in randomisation and ignore inner differentiation. In this context we particularly bring psycho-neurobiology, psycho-immunology and psychosocial epigenetics into play.

Our Chinese arts-therapeutic psycho-oncological research not only deals with the phenomenon of subjective relief but also focuses on underlying mechanisms and the interactive system of aesthetic experience, mental processes and oncological states, and the advanced interdisciplinary domain dealing with cancer pain can serve as a reference-model. There is evidence that cancer pain is distinct from inflammatory pain and neuropathic pain. Specific interactivities between the neoplasm and neurones became evident and translational studies recognise that cancer pain in patients is intimately tied to other symptoms, including anxiety, depression, and sleep deprivation. These form symptom clusters, and somatic and limbic systems converge and affect pain processing [38].

Such approaches cast light on the complex developmental and therapeutic mechanisms in cancer and from the perspective of translational oncology Boilly [39] suggested a comprehensive and holistic approach, which is akin to our Chinese arts-based psycho-oncological activities: 'it is important to broaden the perspective of cancer research by breaking the artificial walls with other disciplines ... incorporating ideas from development, regeneration, stem cells, and neuroscience ... the notion that cancer and normal stem cells are innervated, and thus communicate with the nervous system, raises a larger question, which is the possibility of a higher-order control of cancer growth and regeneration, and possibly integration with sensory input' - and this is precisely what we are dealing with in psycho-oncology: the sensory input of music and the arts.

Cancer immunology [40] has become an important discipline and is inextricably linked with translational medicine. Cancer immunotherapy is one of its most important applications and researchers [41] highlighted an important perspective of scientific success in translational sciences: creativity - and this is also a hallmark of our Chinese approach. Considering that the immune system and the psycho-neural system are closely intertwined - this is the core of psychoneuro and psycho-neuro-endocrine immunology [42] - and that music exhibits psycho-neuro-immunological effects [43] encourages music therapy in oncological and psychooncological fields.

An relatively early Spanish study [44] refers to effects of the immune system on cancer development in rodents subjected to stress and as a result of their research the authors concluded that 'music reduced the suppressive effects of stress on immune parameters in mice and decreased the enhancing effects of stress on the development of lung metastases provoked by carcinosarcoma cells. Music enhanced the immune parameters and the anti-tumor response in unstressed rodents ... music can effectively reverse adverse effects of stress on the number and capacities of lymphocytes that are required for an optimal immunological response against cancer in rodents'. Concerning our Chinese activities on music-psycho-oncology, such results encourage translational studies which combine inferential syntheses and psycho-oncological experiments.

Exploring such complex processes from an interdisciplinary perspective is scientifically extremely challenging and requires further research, which also comprises the still enigmatic profound relationship between the human psyche and the central nervous system. Facing the 2500 years old bodymind-problem, which inheres in core issues of oncological psychosomatics, Mastnak [45] suggested a novel model of sub-atomic (quantum) spheres which incorporate features of both mind and matter and show similarities with the Chinese principle of Qi, one of the cornerstones of Chinese Medicine (TCM). These approaches are far beyond mere input-outputresearch, which is important for the assessment of effect sizes but cannot elucidate the nature of pathogenesis and therapeutic dynamics.

Particularly with regard to arts-based therapies oncological epigenetics come into play. Broadly speaking, epigenetic mechanisms such as DNA methylation, histone modification, nucleosome remodelling and RNA-mediated targeting regulate processes that are fundamental to the genesis of cancer [46], and specific studies cover a wide range from epigenetics in cancer stem cells [47] to breast cancer and the epigenetic factors of its ethology [48].

And here in lies a core of our Chinese translational oncological project. Epigenetics suggest [49] that epigenetic and genetic alterations contribute to cancer initiation and progression, alongside heritable changes in gene expression without alterations in DNA sequences. Disruptions in epigenetic processes can lead to altered gene function and cellular neoplastic transformation, and epigenetic modifications precede genetic changes and usually occur at an early stage in neoplastic development.

Compared with the DNA, epigenetic mechanisms are more flexible and inextricably linked with our socio-cultural life experiences [50]: 'culture acts as another tuneable epigenetic catalyst that both directs developmental trajectories, and becomes convoluted with individual ontology, via a mutuallyinteracting crosstalk mediated by a social interaction that is itself culturally driven ... The cultural and epigenetic systems of heritage may thus provide the "missing" heritability of complex diseases that is currently the subject of much scientific discourse'. Although music-epigenetics is still a fairly undiscovered field, Brigati [51] pointed out that music is a highly transmissible cultural product and social epigenetics describe how communication and emotion - 'prime hallmarks of music' - can be linked to a transmissible, biochemical change, and recent epigenetic studies [52] showed that music listening regulates human microRNA expression. 
Harmonising all these element allows us to draft a multidimensional theoretical framework of music- and artsbased psycho-oncology as a plausible starting point of further interdisciplinary translational psycho-oncological research, as well as practice in clinical contexts and public health.

\section{Multimodal Systems}

Although we fully recognise the research merits of evidence based medicine in the sense of randomised controlled trials and related meta-syntheses, we have to estimate and re-assess their epistemological value in an appropriate way, as well as the model of truth inhering in these modes of research. At the same time we warn about the threat of reductionism in medical sciences and the belief in models which do not mirror the research objects such as how psycho-oncological interventions influence the whole personality.

In this context we suggest a pragmatic four-world-model which must not be confused with multiple-world-models in philosophy such as Karl Popper's [53] Three Worlds Ontology. These four worlds are (i) the material world which concerns both our body and material environments. It comprises endogenous objects such as neurotransmitters, objects that may enter our bodies such as viruses, objects that penetrate our bodies such as radiation and omnipresent 'objects' such as quantum fields; (ii) the mental world, which contains all psychological processes and states, (iii) the sociocultural world, which is inextricably linked with acculturation and hermeneutic understanding and (iv) possible spiritual worlds, which form the core of religions but cannot (if they really exist) be accessed by means of conventional scientific methods.

These worlds are of different quality, inter-connected and form dynamic systems, which are contradictory to scientific assumptions of stability. We suggest that psycho-oncology and arts-based psycho-oncology in particular - should take the inner dynamics of these structures into consideration. By way of illustration: According to genetics and particularly to epigenetics the DNA is not a completely stable structure but contains 'instable' elements such as the transposons, the 'jumping genes', as well as components which can be 'switched on and off'. Particularly newer research associated LINE-1 activity with regulation in cancer [54] and Burns [55] explained that LINE-1 sequences are self-propagating, protein-coding retro transposons, and that their activity results in somatically acquired insertions in cancer genomes: 'altered expression of transposable elements and animation of genomic LINE1 sequences appear to be hallmarks of cancer, and can be responsible for driving mutations in tumorigenesis'.

Taking Daoistic views of permanent changes, which also concern music and the psyche, into account and merge them with epigenetic considerations about the influence of music on gene expression, e.g. that listening to music regulates human microRNA-expression [56], there are good reasons to assume complex dynamic functions of music in oncology and to suggest related translational research. In this context we also draw attention to the fact that miR-132, which belongs to the 'music-sensitive' microRNAs, plays a critical role in regulating TAU protein levels, hence its importance for preventing TAU protein aggregation in Alzheimer's disease. And here in lies perhaps one of the deeper reasons why music therapy is successful in patients with Morbus Alzheimer.
Regarding mathematically formalised models in evidence based medicine such as t-test or Cohen's d, questions about adequate formalisation of such complex systems arise. We do not think that there is a possibility to design a holistic formalised model of our pluralistic world model, but we suggest the introduction of mathematical function with specific arguments, e.g. codes concerning depressive states, aesthetic experiences and chemotherapeutic interventions in the function's domain, and entities such as tumour markers or spiritual experiences in the co-domain. Akin to confidence intervals in classical inferential statistics we suggest confidence zones of the functions which describe tendencies of psychooncologic ally relevant dynamic interdependencies in certain patient populations. This approach significantly differs from the use of statistics in conventional evidence based medicine and also involves mathematical disciplines such as function theory and models which remind in a sense of Heisenberg's uncertainty principle.

\section{Chinese Music Psycho-Oncology: Interdisciplinary Generation of Models}

Musik philosophy poses the ontological question 'What is music?' and there are three contradictory responses: (i) music is the product of the human act of composing and has, once written, its autonomous existence, and this is often the guiding view in musicology and music theory, (ii) music is based on aesthetic experience and has to be seen as an interactive phenomenon, and this is a current opinion in music psychology, particularly in cognitive psychology and neuropsychology of music, (iii) music is a universal micro- and macro-cosmic principle and what we hear is one of its audible (singular) realisations. This position inheres in many myths and traditional philosophies, also in China. This ontological question is also of crucial relevance to our research in Chinese music-psycho-oncology.

Regarding the history of modern (Western) music therapy [57], many models seem to be based on reflective practice, which has become an important tool for the generation of knowledge [58], and subjective theories, which also have gained ground in individualised oncology and psycho-oncology [59]. But they had not been generated through research processes as we know them in pharmacological or surgical research. In Chinese arts-based and music psycho-oncology, however, we emphatically suggest scientific substantiation. Different from primarily physiological agents such as chemotherapy, music in therapeutic context involves a broad spectrum of aesthetic, cultural, psychological, neuro-cognitive, epigenetic and micro-biological factors, which require interdisciplinary research designs to develop and to assess music therapeutic methods in psycho-oncology.

Recognition of the complex influences of music therapy on cancer patient is a crucial issue and we have to be aware of the risks of reductionism and the related spectrum of traps is broad. Also in China we often meet the opinion that a patient's subjective preferences were a reliable criterion to select appropriate music for therapy, which cannot be taken for granted. And rather often we find quantitative measures and the indication of effect sizes without appropriate prestudies whether the applied inventories adequately mirror essential effects or rather yield biased and/or untypical 
data. In this context we firmly suggest to involve critical epistemological analyses and to not believe that satisfying formal methodological requirements of standardised research designs were sufficient for adequate knowledge generation, hence the necessity to generate data on the basis of metatheoretical, epistemological and interdisciplinary musicpsycho-oncological considerations.

Music and the cancer patient form a new entity which is akin to what one of the founders and pioneers of Intermodal Expressive Therapy, Paolo Knill, who passed away in September 2020, called 'the Third' [60]. This 'Third' is not a constant and invariable entity and adequate music-psychooncological research has to take three dynamic modes into consideration: (i) the dynamics of interdependencies between music and the patient, (ii) chronobiology, which is also called the 'fourth dimension in medicine' [61] and which has become a promising perspective in cancer treatment [62] and (iii) the dynamics of clinical settings and socio-cultural public health conditions.

These multiple dynamic conditions, which play a crucial role in translational medicine, qualify the value of scientific outcomes of such types of research which rather stick to inflexible theoretical frameworks than taking epistemological perspectives into account. The seminal discovery of Einstein's theory of relativity, which has also inspired medical, e.g. epidemiological, reasoning [63], springs to mind. In the same way as theory of relativity and quantum field theory fundamentally changed natural scientific paradigms and put classical physics into perspective, systemic dynamic approaches in medical sciences are likely to make linear and one-dimensional research models obsolete.

In this context, feedback-based adjustment and readjustment of appropriate treatment, in our case music psychooncology, is important and three practical domains come into play: (i) continuous optimisation of music therapeutic models and their adjustment for individualised psycho-oncology, (ii) dynamic implementation in clinical practice and (iii) dynamic implementation in public health. In contrast to the use of rigid black-box models to assess mono-dimensional input-outcomecorrespondence, these processes require appropriate research methods such as modified forms of action research, which has also influenced epistemologically oriented mixed-methods research in medicine [64] and inspired dynamic research in multi-dimensional health care $[65,66]$.

From the perspective of theory of science things become complicated and complex. And at this point a key principle of translational medicine afresh comes into play: 'benchto-bedside'. Although we have to spare no effort to satisfy highest epistemological and scientific requirement, there is still the core of our engagement: the individual, mindful and empathetic psycho-oncological work with the cancer patient. And this gap is challenging.

\section{Perspective}

Instead of a conclusion a 'perspective', a term that is sometimes used in novel programmatic and still widely hypothetical medical, also translational scientific, fields. With Chinese artsbased psycho-oncology we not only open new approaches in culturally sensitive, mindful and systemic psycho-oncology.
We also suggest theoretical models which are not consistent with research conventions in evidence based medicine. Looking back to the history of medicine it is very likely that we will come across with obstacles and misunderstandings. Hypotheses and preliminary findings will be criticised as they do not provide classical parameters such as effect sizes or statistical power, although these are not compatible with the basic principle of complex dynamic systems and functional interdependencies of their components. Nonetheless we are convinced that efforts to create models which converge towards the nature of what they try to represent will greatly gain momentum and open minded, critical and interdisciplinary translational research is needed for a flourishing future.

\section{References}

1. Geoff Watts G. Jimmie Coker Holland. Lancet. 2018; 391: 10120.

2. Holland JC. History of psycho-oncology: overcoming attitudinal and conceptual barriers. Psychosom Med. 2002; 64: 206-221.

3. Dolbeault S, Szporn A, Holland JC. Psycho-oncology: where have we been? Where are we going? Eur J Cancer. 1999; 35: 15541558 .

4. Holland JC. Psycho-oncology: Overview, obstacles and opportunities. Psycho oncology. 2018; 27: 1364-1376.

5. Breitbart WS, Alici Y. Psycho-oncology. Harv Rev Psychiatry. 2009; 17: 361-376.

6. Xia J, Merinder LB, Belgamwar MR. Psycho education for schizophrenia. Cochrane Database Syst Rev. 2011.

7. Mastnak W, Tièschky T. Musik-Psycho edukation. Pädagogische und psychiatrische Perspective. Discussion Musikpädagogik. 2017; 75: 5-8.

8. Wilson L, Crowe M, Scott A, et al. Psycho education for bipolar disorder: a discourse analysis. Int J Ment Health Nurs. 2018; 27 : 349-357.

9. Carlson LE. Mindfulness-based interventions for coping with cancer. Ann N Y Acad Sci. 2016; 1373: 5-12.

10. Rubio DM, Schoenbaum EE, Lee LS, et al. Defining translational research: implications for training. Acad Med. 2010; 85: 470-475.

11. Fu Z, Zhang R, Li P, et al. Translational epidemiology: The powerful tool for precision cancer medicine. J Cancer Res Ther. 2019; 15: 269-271.

12. Mastnak W. Systemic Meta-Synthesis. Methodological Innovations [in preparation]. 2021.

13. Krebber AM, Buffart LM, Kleijn G, et al. Prevalence of depression in cancer patients: a meta-analysis of diagnostic interviews and self-report instruments. Psycho oncology. 2014; 23: 121-130.

14. Caruso R, Nanni MG, Riba M, et al. Depressive spectrum disorders in cancer: prevalence, risk factors and screening for depression: a critical review. Acta Oncol. 2017; 56: 146-155.

15. Tsaras K, Papathanassiou IV, Mitsi D, et al. Assessment of Depression and Anxiety in Breast Cancer Patients: Prevalence and Associated Factors. Asian Pac J Cancer Prev. 2018; 19: 16611669.

16. Jia Y, Li F, Liu YF, et al. Depression and cancer risk: a systematic review and meta-analysis. Public Health. 2017; 149: 138-148.

17. Pinquart M, Duberstein PR. Depression and cancer mortality: a meta-analysis. Psychol Med. 2010; 40: 1797-1810.

18. Sotelo JL, Musselman D, Nemeroff C. The biology of depression in cancer and the relationship between depression and cancer progression. Int Rev Psychiatry. 2014; 26: 16-30.

19. Pitman A, Suleman S, Hyde N, et al. Depression and anxiety in patients with cancer. BMJ. 2018; 361: 1415.

20. Linden W, Vodermaier A, Mackenzie R, et al. Anxiety and 
depression after cancer diagnosis: prevalence rates by cancer type, gender, and age. J Affect Disord. 2012; 141: 343-351.

21. An E, Lo C, Hales S, et al. Demoralization and death anxiety in advanced cancer. Psycho oncology. 2018; 27: 2566-2572.

22. Curran L, Sharpe L, Butow P. Anxiety in the context of cancer: A systematic review and development of an integrated model. Clin Psychol Rev. 2017; 56: 40-54.

23. Cororve Gingeret M, Teo I, Epner DE. Managing body image difficulties of adult cancer patients. Cancer. 2014; 120: 633-641.

24. Schover LR. Sexual quality of life in men and women after cancer. Climacteric. 2019; 22: 553-557.

25. Streicher L, Simon JA. Sexual Function Post-Breast Cancer. Cancer Treat Res. 2018; 173: 167-189.

26. Wang K, Ariello K, Choi M, et al. Sexual healthcare for cancer patients receiving palliative care: a narrative review. Ann Palliat Med. 2018; 7: 256-264.

27. Lina Mahayati S, Allenidekania Happy H. Spirituality in adolescents with cancer. Enferm Clin. 2018; 28: 31-35.

28. Paredes AC, Pereira MG. Spirituality, Distress and Posttraumatic Growth in Breast Cancer Patients. J Relig Health. 2018; 57: 16061617.

29. Feuchtwang S, (ed). Handbook on Religion in China. Cheltenham, UK: Edward Elgar Publishing 2020.

30. McLean LM, Nissim R. Marital therapy for couples facing advanced cancer: case review. Palliat Support Care. 2007; 5: 303-313.

31. Naaman S, Radwan K, Johnson S. Coping with early breast cancer: couple adjustment processes and couple-based intervention. Psychiatry. 2009; 72: 321-345.

32. Nakaya N. Effect of Psychosocial Factors on Cancer Risk and Survival. J Epidemiol 2014; 24: 1-6.

33. Ahn HK, Bae JH, Ahn HY, et al. Risk of cancer among patients with depressive disorder: a meta-analysis and implications. Psycho oncology. 2016; 25: 1393-1399.

34. Lutgendorf SK, DeGreest K, Dahmoush L, et al. Social isolation is associated with elevated tumor norepinephrine in ovarian carcinoma patients. Brain Behav Immun. 2011; 25: 250-255.

35. Mravec B, Horvathova L, Hunakova L. Neurobiology of Cancer: the Role of $\beta$-Adrenergic Receptor Signalling in Various Tumor Environments. Int J Mol Sci. 2020; 21: 7958.

36. Lamboy-Caraballo R, Ortiz-Sanchez C, Acevedo-Santiago A, et al. Norepinephrine-Induced DNA Damage in Ovarian Cancer Cells. Int J Mol Sci. 2020; 21: 2250.

37. Acheampong T, Jiang L, Ziogas A, et al. Multi-Systemic Biological Risk and Cancer Mortality: The NHANES III Study. Sci Rep. 2020; 10: 5047.

38. Schmidt BL. The neurobiology of cancer pain. Neuroscientist. 2014; 20: 546-562.

39. Boilly B, Faulkner S, Jobling P, et al. Nerve Dependence: From Regeneration to Cancer. Cancer Cell. 2017; 31: 342-354.

40. Rezaei N. Cancer Immunology: A Translational Medicine Context. Cham, CH: Spinger. 2020.

41. Miller JF, Sadelain M. The journey from discoveries in fundamental immunology to cancer immunotherapy. Cancer Cell. 2015; 27: 439-449.

42. França K, Lotti TM. Psycho-Neuro-Endocrine-Immunology: A Psychobiological Concept. Adv Exp Med Biol. 2017; 996: 123134.

43. Fancourt D, Ockelford A, Belai A. The psycho neuro immunological effects of music: a systematic review and a new model. Brain Behav Immun. 2014; 36: 15-26.
44. Núñez MJ, Mañá $P$, Liñares $D$ et al. Music, immunity and cancer. Life Sci. 2002; 71: 1047-1057.

45. Mastnak W. Subatomare Bewusstheit und Musiktherapie. Quantenphysikalische Hypothesen zur psychosomatischen Wirkung von Musik. Musik- Tanz und Kunsttherapie. 2013; 24: 174-187.

46. Dawson MA, Kouzarides T. Cancer epigenetics: from mechanism to therapy. Cell. 2012; 150: 12-27.

47. Toh TB, Lim JJ, Chow EK. Epigenetics in cancer stem cells. Mol Cancer. 2017; 16: 29.

48. Wu Y, Sarkissyan M, Vadgama JV. Epigenetics in breast and prostate cancer. Methods Mol Biol. 2015; 1238: 425-466.

49. Kanwal R, Gupta K, Gupta S. Cancer epigenetics: an introduction. Methods Mol Biol. 2015; 1238: 3-25.

50. Wallace R, Wallace D, Priami C, et al. Cultural Epigenetics: On the Heritability of Complex Diseases. Transactions on Computational Systems Biology XIII. Lecture Notes in Computer Science. 6575. Berlin, Heidelberg: Springer. 2011; 131170 .

51. Brigati C, Saccuman MC, Banelli B, et al. Toward an epigenetic view of our musical mind. Front Genet. 2012; 2: 111.

52. Nair PS, Raijas P, Ahvenainen M, et al. Music-listening regulates human microRNA expression. Epigenetics. 2020; 6: 1-13.

53. Popper K. Objective Knowledge: An Evolutionary Approach. Oxford: Oxford University Press. 1979.

54. Rodic N. LINE-1 activity and regulation in cancer. Front Biosci. 2018; 23: 1680-1686.

55. Burns KH. Transposable elements in cancer. Nat Rev Cancer. 2017; 17: 415-424.

56. Nair PS, Raijas $P$, Ahvenainen $M$, et al. Music-listening regulates human microRNA expression. Epigenetics. 2020; 6: 1-13.

57. Mastnak W. The Evolution of Music Therapy. Five Eras and Their Spirit. Musik- Tanz und Kunsttherapie. 2015; 26: 206-220.

58. Thompson N, Pascal J. Developing critically reflective practice. Reflective Practice. 2012; 13: 311-325.

59. Koehler M, Koenigsmann M, Frommer J. Coping with illness and subjective theories of illness in adult patients with haematological malignancies: systematic review. Crit Rev Oncol Hematol. 2009; 69: 237-257.

60. Knill P, Peterson P. Das unvermittelbare Heilmittel oder das Dritte in der Kunsttherapie. Ansätze kunsttherapeutischer Forschung. Berlin: Springer Verlag. 1990.

61. Cederroth CR, Albrecht U, Bass J, et al. Medicine in the Fourth Dimension. Cell Metab. 2019; 30: 238-250.

62. Sulli G, Lam MTY, Panda S. Interplay between Circadian Clock and Cancer: New Frontiers for Cancer Treatment. Trends Cancer. 2019; 5: 475-494.

63. Garira W. The Replication-Transmission Relativity Theory for Multi scale Modelling of Infectious Disease Systems. Sci Rep. 2019; 9: 16353.

64. Sendall MC, McCosker LK, Brodie A, et al. Participatory action research, mixed methods, and research teams: learning from philosophically juxtaposed methodologies for optimal research outcomes. BMC Med Res Methodol. 2018; 18: 167.

65. Bradbury H, Lifvergren S. Action research healthcare: Focus on patients, improve quality, drive down costs. Health Manage Forum. 2016; 29: 269-274.

66. Cordeiro L, Soares CB. Action research in the healthcare field: a scoping review. JBI Database System Rev Implement Rep. 2018; 16: 1003-1047. 